\title{
Bloqueos de extremidad inferior
}

\section{Lower extremity blocks}

Daniela Bravo MD. ${ }^{1}$, Julián Aliste MD. ${ }^{1}$, Sebastián Layera MD. ${ }^{1}$, Álvaro Jara MD. ${ }^{1}$

\begin{abstract}
Lower limb blocks are described with emphasis on distribution according to sensitive distribution and its sonoanatomy.

Términos Mesh: lower extremity blocks.

\section{RESUMEN}

Se describen los bloqueos de la extremidad inferior haciendo énfasis en la distribución de acuerdo a distribución sensitiva y a su sonoanatomia.
\end{abstract}

\section{Key words:}

Lumbar plexus blocks, sacral plexus blocks, nerve blocks

Palabras clave:

Bloqueos de plexo lumbar, bloqueos de plexo sacro, bloqueos de nervio periférico, extremidad inferior, anestesia regional.

\section{Introducción}

A diferencia de su contraparte superior, la extremidad inferior no puede ser bloqueada mediante una técnica periférica única, dado que es inervada por dos plexos (lumbar y sacro) que se encuentran los suficientemente separados para no ser alcanzados en un abordaje único, contrastando así con las eficaces y eficientes técnicas neuroaxiales. Para lograr un efecto clínico potencialmente equivalente, ambos plexos deben ser bloqueados a nivel proximal, lo que se traduce en punciones profundas, resultando en las mismas precauciones de complicaciones hemorrágicas que una punción neuroaxial y, que por otra parte, requieren grandes masas de droga para optimizar latencia y densidad de bloqueo, pudiendo desencadenar toxicidad por anestésicos locales. Es por todo esto que los bloqueos de extremidad inferior continúan siendo alternativas más bien analgésicas, pero que mediante el mejor entendimiento de la anatomía y el uso ultrasonografía han crecido en variedad y perfiles de desempeño.

\section{Conceptos anatómicos relevantes}

\section{Plexo lumbar}

Este plexo está conformado por fibras de los nervios espinales L1-L3 con aportes variables de T12 y L4 (Figura 1). Tras su emergencia a través de los forámenes intervertebrales, los ramos ventrales de las raíces involucradas se sitúan en el espesor del músculo psoas por delante de las apófisis transversas. Aquí se dividen en un componente anterior y posterior, resultando de su interrelación los nervios terminales de este plexo, que descienden junto al músculo y van emergiendo

Departamento de Anestesiología y Medicina Perioperatoria, Universidad de Chile.

Fecha de recepción: 01 de diciembre de 2019

Fecha de aceptación: 01 de diciembre de 2019

\section{ORCID}

https://orcid.org/0000-0003-0611-3623

Correspondencia:

Daniela Bravo MD

Email: dbravoadvis@uchile.cl 


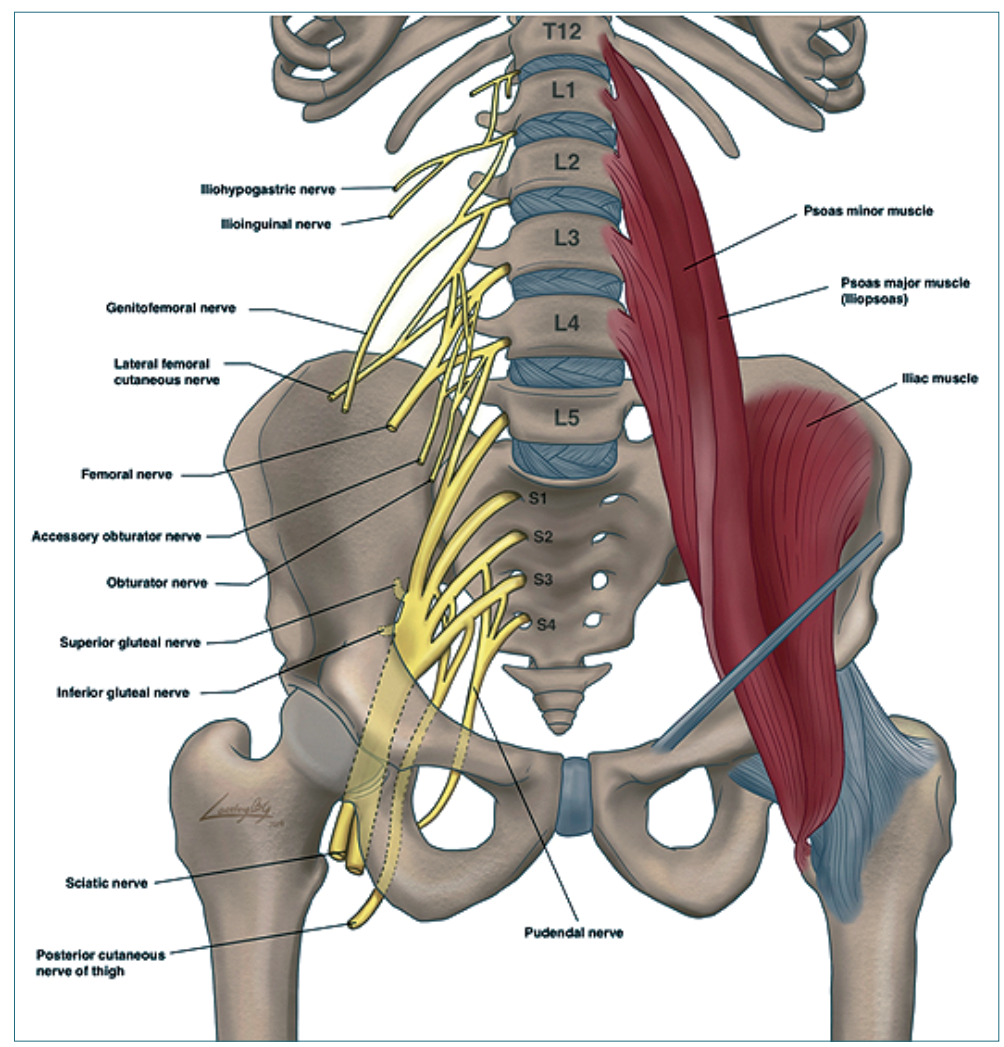

Figura 1. Plexo lumbosacro.

hacia lateral y caudal[1],[2]. Desde su origen, todos los nervios se encuentran bajo la misma cobertura, la fascia iliaca (FI).

Los nervios iliohipogástrico e ilioinguinal se originan principalmente de la raíz L1 y el nervio genitofemoral de L2[1]. Estos tres componentes del plexo lumbar van a contribuir a la inervación de la piel y musculatura de la pared abdominal distal, y zonas proximales de la extremidad inferior y genitales[3].

Los nervios terminales principales de la extremidad inferior son el cutáneo femoral lateral (NCFL) (L2-L3), puramente sensitivo y, femoral (NF) (L2-L3-L4) y obturador (NO) (L2-L3-L4), ambos mixtos. A nivel lumbar, estos nervios se encuentran en íntima relación con el borde posterior del músculo psoas, donde la distancia habitual desde la apófisis transversa de $L 4$ al plexo no supera los 2,5 cm[4],[5]. Cuando entran en la pelvis, si bien se mantienen bajo la misma fascia, se separan siguiendo trayectorias diferentes y ya a nivel inguinal los tres nervios se encuentran se encuentran francamente separados[1].

El NCFL, tras emerger desde psoas, avanza en dirección a la espina iliaca antero-superior (EIAS), donde se superficializa y abandona la pelvis medial a ésta, bajo el ligamento inguinal (LI)[6]. A nivel inguinal se ubica superficial a la Fl en relación con la cara anterior del músculo sartorio para luego posicionarse en un túnel delimitado por la fascia lata entre el sartorio y el tensor de la fascia lata[7].

El NF es el componente más relevante del PL. Tiene un trayecto más bien vertical, emergiendo del psoas en un plano posterior y luego sigue hacia caudal en un surco entre el psoas y el músculo iliaco, manteniéndose bajo la FI. Ingresa al triángulo femoral (TF) del muslo tras pasar bajo el LI. A este nivel se encuentra 1 a $2 \mathrm{~cm}$ lateral al paquete vascular femoral[8], del cual se halla separado por la cintilla iliopectínea. El TF constituye una entidad triangular de base superior formada por el LI, delimitada por lateral por el músculo sartorio (MS) y el por medial por el músculo aductor largo (MAL), siendo el vértice el punto de intersección de los bordes mediales de estos. Salvo por las ramas al músculo iliaco y las articulares que se dirigen a la cadera, el NF tiende a dividirse en el TF entre $\mathrm{LI}$ y pliegue inguinal (aproximadamente a $3 \mathrm{~cm}$ del primero) para formar sus ramos terminales, los que permanecen inmersos en tejido conectivo extraneural común y que eventualmente perforan la Fl para dirigirse a sus objetivos. Algunos de 
estos nervios, como el nervio al músculo vasto medial (NMVM) y nervio safeno (NS), continúan distalmente con los vasos femorales bajo en MS una vez que emergen del TF. El canal aductor (CA) constituye un túnel subaponeurótico que se origina en el vértice del TF y culmina en el hiato aductor. Posteromedialmente está delimitado por los músculos aductores (largo y magno), anterolateralmente por el músculo vasto medial y anteriormente por la membrana vastoaductora (MVA), ubicada profunda al MS. En su interior transcurren los vasos femorales superficiales y el NS. Por su parte, el NMVM tiene también un trayecto subsartorial, pero se encuentra en un compartimento miofascial distinto pero adyacente al CA. Entre la MVA y el MS, el NS junto al nervio cutáneo medial y la división anterior del NO forman el plexo subsartorial[2]. Luego, el NS emerge por anterior del CA perforando la MVA, participando en la inervación perigenicular (junto al NO)[9],[10] y de la pierna. Así, el nervio femoral entrega inervación articular (cadera, rodilla, tobillo y talocalcaneonavicular), ósea (pelvis, fémur y tibia), muscular (pectíneo, iliaco y componentes del compartimento anterior del muslo), y cutáneo (piel antero-medial del muslo, rodilla, y cara medial de la pierna, tobillo y pie).

El NO tiene una posición y trayecto más medial. Emerge por posterior del psoas descendiendo verticalmente hacia el foramen obturador de la pelvis, para dirigirse hacia el compartimento medial del muslo, ubicándose de manera inicial entre los músculos obturador externo y pectíneo, para luego posicionarse entre los aductores cuando ya se ha dividido[2]. Es frecuente su anastomosis con ramas provenientes de otros nervios contribuyendo a distintos territorios. El nervio obturador inerva articulaciones (parte de la cadera y rodilla), huesos (porciones pélvicas y de fémur), musculatura (compartimento aductor del muslo), y un territorio cutáneo variable (muslo póstero-medial). La existencia del nervio obturador accesorio (NOA) (L3L4) es variable (8-54\%), y su presencia es relevante desde el punto de vista anestésico regional ya que inerva consistentemente al músculo pectíneo y la cápsula anterior de la cadera, pero posee un trayecto diferente al NO una vez que ha dejado el psoas, siendo por anterior a la eminencia iliopúbica y no a través del agujero obturador[11].

\section{Plexo sacro}

Este plexo está conformado por la unión del tronco lumbo-sacro (que proviene de los ramos ventrales de las raíces de L4-L5) y las raíces S1-S4. Se forma en la pelvis anterior al sacro y músculo piriforme, y posterior a la fascia presacra[2]. De sus 12 nervios terminales principales, los relevantes para las técnicas regionales de la extremidad inferior son los nervios ciático (NC) y cutáneo femoral posterior (NFCP).

Clínicamente, ante procedimientos de extremidad inferior, el NC es el más importante de este plexo, ya que inerva porciones articulares (cadera, rodilla y tobiIlo), óseas (pelvis, fémur, tibia, peroné y del pie), músculos (compartimento posterior del muslo, la de la pierna y pie), y piel (aspecto posterior del muslo, casi toda la pierna y pie). Está conformado por dos componentes desde su origen; el nervio tibial (NT) (L4-S3), más grueso y anterior, y el nervio peroneo (NP) (S1S3)[2]. Ambos nervios tienen un trayecto común, pero no entremezclan sus fibras[12]. Una vez formado, el NC abandona la pelvis a través del agujero ciático mayor entrando en la zona glútea, donde queda anterior al músculo piriforme para luego continuar el trayecto caudal hacia el muslo, ubicándose inicialmente entre el trocánter mayor y la tuberosidad isquiática, profundo al músculo glúteo mayor y caudal al músculo piriforme[2]. En el primer tercio del muslo tiene una posición medial al fémur la cual se hace lateral en su tercio distal con una relación lateral y posterior a los vasos poplíteos[2]. En la mayoría de los casos, al entrar en la fosa poplítea se divide en sus componentes tibial (medial) y peroneo (lateral), sin embargo, esto también puede ocurrir en cualquier punto de su trayecto hasta la rodilla. En esta zona, ramas del nervio tibial junto a ramas de la división posterior del NO forman el plexo poplíteo posterior[9],[10],[13].

El NCFP (S1-S3), es un nervio puramente sensitivo. Emerge de la pelvis por el agujero ciático mayor, medial al NC, ubicándose anterior al glúteo máximo, pero dentro de su fascia, y adyacente a los vasos glúteos inferiores. Una vez en el muslo, se ubica en un plano fascial superficial, que lo separa del nervio ciático, desde donde continúa subcutáneamente hacia caudal hasta la pantorrilla. Inerva las regiones cutáneas glútea inferior, parte del perineo, la cara posterior del muslo (incluyendo la zona poplítea) y de la pierna[2].

\section{Regiones de la extremidad inferior}

Al igual que en la extremidad superior, la cobertura del periostio es fundamental para el manejo analgésico (Figura 2), siendo también importante el conocimiento de la inervación de la piel y grupos musculares al realizar bloqueos anestésicos y fundamentales para poder evaluar el éxito de un procedimiento.

Cirugía de cadera y fémur proximal/medio: los bloqueos útiles son lo que aborden los plexos o 


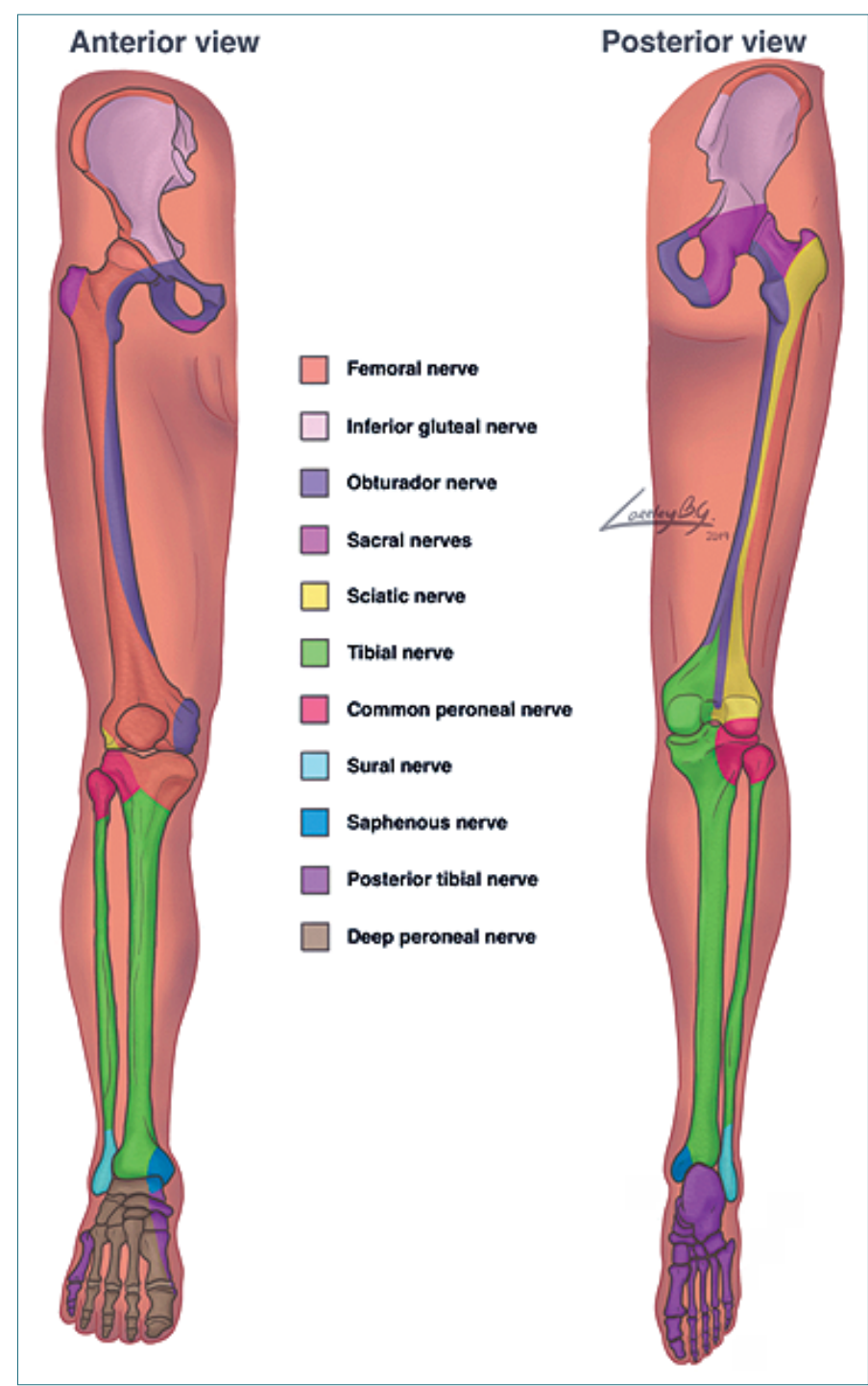

Figura 2. Inervación ósea extremidad inferior. Visión anterior y posterior. nervios terminales a poco de su formación, de manera tal de cubrir las ramas que emergen precozmente; del PL lo sería el bloqueo del compartimento del psoas (o equivalente), y del PS los abordajes parasacro o transglúteo.

Cirugía de fémur medio/distal: son útiles todos los abordajes que bloqueen el NF Y NO desde el psoas hasta el vértice del TF o ingle respectivamente, y los bloqueos proximales del NC (subglúteo y anterior clásico).

Cirugía de rodilla: se emplean bloqueos del NF desde inguinal hasta el canal aductor, bloqueos del NC sobre la rodilla (subglúteo, anterior clásico, medio femoral y poplíteo) y del NO que cubran la división posterior.

Cirugía de pierna: si bien cuestionables ante el riesgo de un síndrome compartimental enmascarado, los bloqueos relevantes son el componente ciático (medio femoral o poplíteo) y bloqueo del NS por sobre la rodilla.

Cirugía de tobillo: bloqueo del NC (poplíteo) y bloqueo del NS (de existir un componente quirúrgico medial).

Cirugía de pie: bloqueo del NC (poplíteo) y NS si hay compromiso medial. En el caso de que la cirugía contemple sólo el antepié podría considerarse un blo- 
queo de tobillo.

Cabe destacar además que la inervación articular cobra especial relevancia debido a que son cirugías frecuentes donde tanto la analgesia como la capacidad de rehabilitación funcional son fundamentales. Es así como en muchos casos se debe privilegiar el componente que más requerimientos otorgue y suplementar con otras alternativas analgésicas el resto, de manera tal de evitar bloqueo motor innecesario, así como también grandes masas de droga. La inervación de la articulación de cadera por su cara anterior está dada por ramas articulares de los NF, NO/NOA, mientras que el componente posterior está provisto por componentes del PS; nervio glúteo superior, nervio al músculo cuadrado femoral y NC[11],[14] sin embargo, en esta zona es donde se encuentran principalmente mecanorreceptores y no nociceptores[15]. En el caso de la rodilla, su cara anterior esta provista por ramos del NF, a través de los nervios al vasto medial (NVM) y NS, y obturador (división anterior)[10],[16] y por posterior, por los NC (ambos componentes) y NO (división posterior)[10],[16].

\section{Bloqueos del plexo lumbar}

Nos referiremos al bloqueo del PL propiamente tal, así como también a los principales bloqueos de sus nervios terminales que se dirigen a la extremidad inferior.

\section{Bloqueo del plexo lumbar}

El bloqueo del plexo lumbar (BPL), también denominado bloqueo del compartimento del psoas, es el abordaje más proximal y único por vía posterior. A nivel lumbar paravertebral los tres nervios que van a la extremidad inferior (NF, NO Y NCFL) pueden ser abordados de manera consistente antes de su ingreso a la pelvis.

Para este abordaje existen diversas técnicas que van desde la pérdida de resistencia al ultrasonido (US).
La neuroestimulación (NE) fue por mucho tiempo la técnica de elección, sin embargo, al ser un bloqueo profundo y dependiente de múltiples y variables reparos de superficie, requiere de experiencia para interpretar las respuestas motoras y evitar complicaciones. Es así como varias técnicas fueron desarrolladas[4], todas con similares tasas de éxito, pero siendo la de Capdevilla la más validada por precisar imagenológicamente el punto de entrada y márgenes de avance[4]. Sin embargo, el US se ha posicionado como la alternativa más confiable en la actualidad ya que permite ver el objetivo. Para su realización, a su vez, hay múltiples técnicas como la del "Tridente"[17], paramediana transversa[18] y la de "Shamrock" o trébol[19],[20], siendo esta última la que se revisará, dado que permite una mejor visualización del psoas y la aguja, lo que se traduce en una ejecución más fácil.

Con el paciente en decúbito lateral con el lado a operar hacia arriba se identifica la línea media y la cresta iliaca. Un transductor convexo de baja frecuencia se posiciona verticalmente entre la cresta iliaca y el reborde costal, programando con una profundidad de mapeo de acuerdo con el hábito corporal (Figura 3A). La imagen a obtener es la del cuerpo vertebral y apófisis transversa de $\mathrm{L} 3$ o L4, asociado a tres masas musculares; el erector de la columna por posterior, el cuadrado lumbar insertándose en la apófisis y el psoas entre el cuerpo vertebral y la apófisis (Figura 3B). En el cuadrante posteromedial del psoas, puede verse una imagen hiperecogénica que suele corresponder al plexo. Resulta habitual ver el polo inferior renal intermitentemente con la respiración justo anterior al psoas. Una vez lograda esta imagen, se puede hacer un sutil desplazamiento o inclinación hacia caudal para desproyectar la apófisis transversa para luego avanzar desde posterior a anterior una aguja de entre 80 y $100 \mathrm{~mm}$. El punto de inserción de la aguja se estima según la profundidad del objetivo en la pantalla, para lograr así un trayecto perpendicular a los haces de ultrasonido. En caso de contactar con la apófisis transversa, esta deberá ser cabalgada para llegar al plexo. El uso de NE concomitante al US era considerado

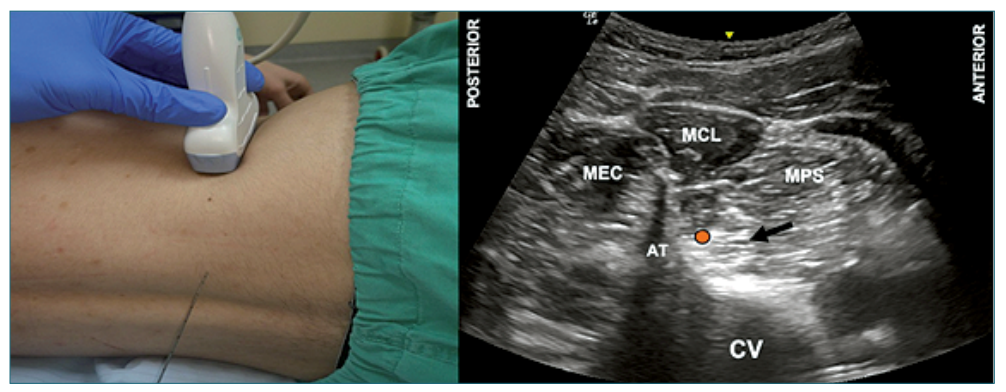

Figura 3. Bloqueo de plexo lumbar (BLP). A. Posición transductor BPL; B. Sonoanatomía BPL: MEC: músculo erector de la columna; MCL: músculo cuadrado lumbar; MPS: músculo psoas; CV: cuerpo vertebral; AT: apófisis transversa; Punto naranjo: punto inyección cuadrante pósteromedial músculo psoas. 
mandatorio hasta hace poco, sin embargo, empleando sólo US (inyectando en el cuadrante posteromedial del psoas) si bien se aumenta la latencia, la eficacia se mantiene[21]. La NE aún puede aportar en casos de dificultad en identificar los reparos ultrasonográficos.

Un estudio de búsqueda de dosis que utilizó el método secuencial de Dixon, estimó por medio de regresión logística una dosis efectiva 95 (DE95) de ropivacaína 0,5\% de $36 \mathrm{~mL}$ (IC 95\% 19,7-52,2 mL)[22].

Una vez alcanzado el objetivo, los catéteres pueden ser avanzados ciegamente, con NE o bajo US, existiendo escasa evidencia que apoye una técnica por sobre otra[2].

Al ser un bloqueo profundo, lo rigen las mismas recomendaciones de manejo de anticoagulación que para el neuroeje[23]. Hay que tener además especial precaución al inyectar ya que este es considerado un sitio de alta absorción sistémica, por lo que se debe procurar no estar intravascular, fraccionar el volumen, emplear un vasoconstrictor y de cuidar la masa de droga total. Se debe contemplar además que el emplear presiones de inyección mayores a 20 psi aumenta la incidencia de difusión peridural[24]. Resulta fundamental vigilar la aparición de complicaciones como punción/difusión espinal o peridural, o hematomas renales, retroperitoneales o del mismo psoas. Asimismo, se debe tener precaución por el riesgo de caídas asociadas al bloqueo del cuádriceps.

\section{Bloqueo 3:1 y Bloqueo de la fascia ilíaca a nivel inguinal}

Ambas técnicas merecen una mención dado que aparecen recurrentemente citadas en la literatura. El objetivo clínico de ambas era lograr, por vía anterior y a nivel inguinal, un equivalente al BPL, sin embargo, ninguno de los dos abordajes ha demostrado cubrir los tres nervios deseados, sino más bien el NF en forma consistente y el NCFL en la mayoría de los casos[25]. Esto se debería a que la difusión del AL no logra ascender lo suficiente para alcanzar el plexo, sino más bien presenta sólo una extensión lateral, lo que explica por qué no se logra alcanzar las ramas articulares a la cadera del NF ni tampoco al NO, que a este nivel se encuentra compartimentado medialmente[25]-[27].

\section{Bloqueo de la fascia ilíaca suprainguinal}

Dado que la técnica original descrita por Dalens sólo logra difusión lateral a nivel inguinal[28], surge la idea de realizar una inyección más cefálica y así facilitar la llegada del AL hacia proximal[29],[30], lo que tendría utilidad en el escenario de cirugía de cadera y podría constituir una alternativa más segura al bloqueo del plexo lumbar. Mediante estudios imagenológicos y cadavéricos se ha podido observar que, si bien el ascenso al origen del plexo no ocurre, si podría lograr una difusión cefálica y medial lo suficiente para alcanzar el NO[30]-[32]. Aún existen limitados estudios que avalan la eficacia del Bloqueo de fascia ilíaca suprainguinal (BFIS) en distintos escenarios clínicos.

Inicialmente descrito como técnica de pérdida de resistencia (doble pop)[29], sin embargo, el abordaje ha prosperado mediante el uso de US[30],[33].

Con el paciente en supino y con extensión de cadera, se emplea un transductor lineal de alta frecuencia que se ubica parasagitalmente medial a la EIAS y perpendicular al LI, con el objetivo de hallar las referencias de interés (Figura $4 \mathrm{~A}$ ); hacia caudal una imagen triangular del extremo proximal del músculo sartorio y hacia cefálico una imagen similar en espejo que corresponde al oblicuo interno. El punto de contacto de ambos músculos genera la característica imagen de corbatín y constituye el punto de profundización hacia la Fl. Justo bajo este plano, se aprecian fibras musculares de aspecto envolvente, el músculo iliaco, sobre un elemento óseo profundo que corresponde al hueso homónimo (Figura 4B). Se avanza una aguja en plano desde latero-caudal a súpero-medial $y$, una vez que se atraviesa la Fl, se confirma el plano mediante hidrodisección, para progresivamente avanzar hacia cefálico en el bolsillo que se va creando, procurando observar la elevación del plano del oblicuo interno y de la arteria circunfleja profunda[30],[33].

El punto de entrada en la piel puede variar se-

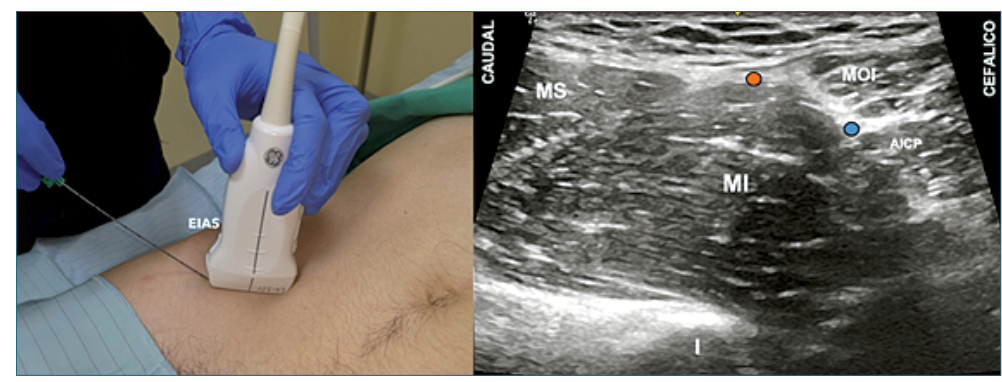

Figura 4. Bloqueo de fascia ilíaca suprainguinal (BFIS). A. Posición transductor BFIS; B. Sonoanatomía BFIS: MS: músculo sartorio; MOI: músculo obturador interno; MI: músculo iliopsoas; I: hueso ilíaco; AICP: arteria circunfleja ilíaca profunda; Punto naranjo: punto de entrada de aguja en fascia ilíaca; Punto azul: sitio de inyección suprainguinal. 
gún la técnica empleada (bajo o sobre el ligamento inguinal) pero el sitio donde la aguja perfora la Fl es el mismo[30],[33].

No existen publicaciones de búsqueda de dosis con metodología óptima, sin embargo, existen estudios imagenológicos que sugieren que el volumen necesario para lograr difusión hacia el nervio obturador es de alrededor de $40 \mathrm{~mL}[31],[32]$.

Si bien la instalación de un catéter es posible, aún no existe evidencia acerca de su efectividad ni experiencia en relación con su manejo y potenciales complicaciones propias.

Existe el riesgo teórico de hematoma o inyección intravascular por punción de la arteria circunfleja profunda, daño del NF si es que no se tiene la precaución de identificarlo previo a la inyección, y de avanzar intraperitoneal y generar una lesión visceral. Además, al contemplar el bloqueo del NF, existe el riesgo inherente de caídas secundarias a bloqueo motor.

\section{Bloqueo de grupo de nervios pericapsulares (Pericapsular nerve group $=$ PENG)}

Esta técnica busca un bloqueo selectivo de las ramas articulares de la cadera que provienen del $\mathrm{PL}$, con el objetivo de evitar el bloqueo motor de otros abordajes[34]. Se basa en la difusión del AL entre el músculo iliopsoas y la cápsula articular. Aún carece de estudios que avalen su eficacia en distintos escenarios clínicos.

La mayoría de las ramas articulares a la cadera del NF emergen antes del LI, avanzan a través del músculo iliaco hacia la cápsula anterior en un plano profundo sobre la superficie del periostio del pubis entre la EIAS y la eminencia iliopúbica[11]. Por su parte, el NOA avanza directamente anterior a la misma eminencia iliopúbica[11]. Sólo las ramas articulares de NO (que nacen antes o al pasar por el agujero obturador) tienen un trayecto en un plano distinto[11].

Constituye es un abordaje eminentemente ultrasonográfico, donde, con el paciente en supino, un transductor curvo de baja frecuencia se posiciona de manera transversa sobre la EIAS, para luego rotar en $45^{\circ}$ para alinearse con la rama púbica (Figura 5A)[34]. Se inserta una aguja de 80-100 mm en plano desde lateral buscando alcanzar el plano entre el tendón del psoas y la rama púbica (Figura 5B).

No existen estudios de búsqueda de dosis. El volumen inicialmente descrito es de $20 \mathrm{ml}$ y si bien es técnicamente factible instalar catéteres, no hay literatura al respecto.

Existe el riesgo teórico de punción vascular y lesión del NF. El riesgo de difusión del AL y posibilidad de bloqueo del NF no está claro.

\section{Bloqueo del nervio femoral}

De los tres nervios a la extremidad inferior éste es el más importante en términos de analgesia bajo la cadera.

Puede ser eficazmente bloqueado guiándose tanto con pérdida de resistencia, parestesias, NE o US dado que su ubicación a nivel inguinal es lateral a la arteria femoral y sólo un plano aponeurótico más profundo. Al comparar US versus el uso conjunto de US más NE o US versus NE no existen diferencias de eficacia, pero el uso de US consume menos tiempo, requiere de menos pases de agujas y permite ahorrar $\mathrm{AL}$.

El bloqueo se realiza con el paciente en decúbito supino y la extremidad inferior extendida. Para la técnica con US se emplea un transductor lineal de alta frecuencia que se ubica paralelamente entre el pliegue y el LI (Figura 6A), donde se debe mapear la zona hacia medial y lateral para localizar los vasos femorales. En este corte el NF se ve en eje corto, lateral a la arteria, como una estructura hiperecoica ovalada o fusiforme, que reposa sobre el músculo iliopsoas y bajo dos líneas que corresponden a las fascias lata e ilíaca (Figura 6B). El nervio no siempre se delimita bien, pero habitualmente sí las estructuras que lo colindan. Una vez identificado ya sea el nervio o su compartimento, se inserta una aguja de $50 \mathrm{~mm}$ a 100 $\mathrm{mm}$ en plano con US desde lateral, con el objetivo de alcanzar el borde lateral o profundo del nervio femoral[3]. Una vez ahí, se inyecta el AL lo que debiese

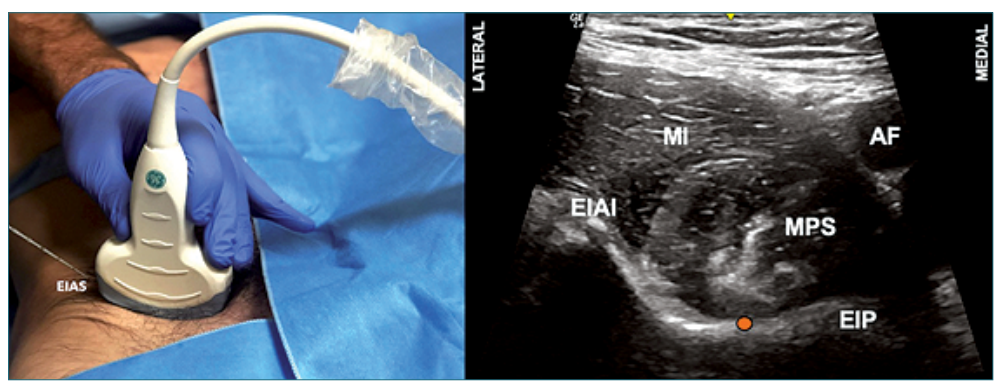

Figura 5. Bloqueo grupo de nervios pericapsulares (PENGB). A. Posición transductor PENGB; B. Sonoanatomía PENGB: MI: músculo ilíaco; MPS: músculo psoas; EIAl: espina ilíaca anteroinferior; EIP: eminencia iliopúbica; AF: arteria femoral; Punto naranjo: sitio de inyección. 


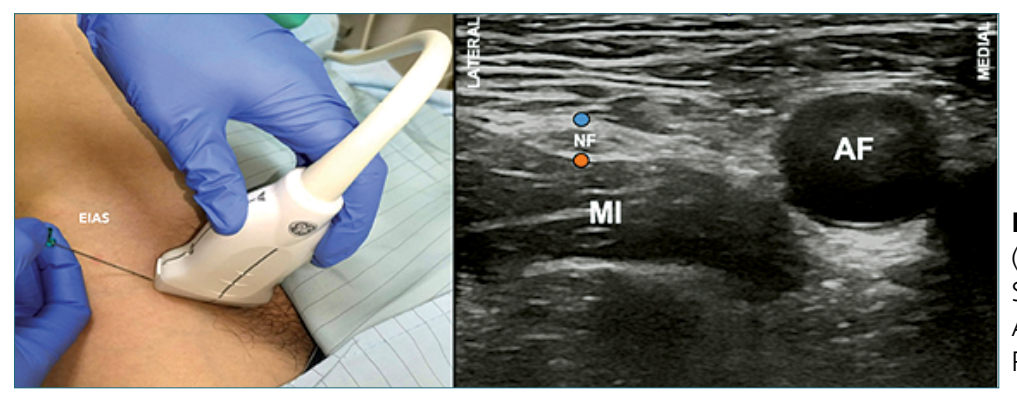

Figura 6. Bloqueo del nervio femoral (BNF). A. Posición transductor BNF; B. Sonoanatomía BNF: MI: músculo ilíaco; AF: arteria femoral; NF: nervio femoral; Punto naranjo y azul: sitios de inyección.

lograr delimitar la estructura, de lo contrario, se debe reposicionar cuidadosamente la aguja para optimizar la difusión.

Mediante un estudio de búsqueda de dosis, se estimó que con US el VEM50 de Ropivacaína 0,5\% es $15 \mathrm{~mL}$ mientras que con NE $26 \mathrm{~mL}$ [35].

Cuando se utiliza NE, obtener respuesta motora del MS o del cuádriceps resulta igualmente efectivo[36], y por otra parte, al emplear US no hay evidencia que avale la necesidad de inyección circunferencial versus parcial alrededor el nervio[3].

Para su instalación, el avance del catéter puede realizarse exitosamente de manera ciega, bajo NE o US. El empleo de catéteres estimulantes parece no aportar un beneficio significativo, pero si se opta por su uso, el US concomitante con la NE para la búsqueda del NF permite acortar el tiempo de instalación[2]. Al emplear US, instalar el catéter perpendicular al NF toma menos tiempo que longitudinalmente, sin haber diferencias en eficacia, y ubicar el catéter profundo al nervio otorga un bloqueo más denso que por enci$\mathrm{ma}[2]$.

Si bien es un bloqueo básico, existe el riesgo de lesión neural y vascular. El riesgo de caídas por bloqueo del cuádriceps es un elemento a tener en consideración.

\section{Bloqueos del nervio femoral a nivel del triángulo femoral y del canal aductor}

Aunque el bloqueo del CA fue inicialmente pensado para bloquear el NS para cirugía de tobillo, actualmente estos abordajes tienen por objetivo alejarse del tronco común del NF y así evitar bloquear todas sus ramas motoras, con lo cual puede preservarse parte de la función muscular del cuádriceps, pero conservando su capacidad analgésica en cirugía de rodilla (por vía anterior)[2]. Si bien en el bloqueo a nivel inguinal el NF también se encuentra en el TF, este término se emplea para los bloqueos que se realizan cercanos a su vértice y en el caso del bloqueo del CA, para los procedimientos más distales al triángulo. En la literatura se ha generado confusión relacionada a estos abordajes ya que por mucho tiempo se estableció como sitio de punción el punto medio del muslo, medido desde la EIAS hasta el borde superior de la patela, lo que en realidad corresponde al TF, y por otra parte, muchas veces se incluía como parte del contenido del CA el NMVM e incluso ramos del NO, los que estrictamente no se encuentran en su interior, pero si en las inmediaciones, por lo que su bloqueo conjunto podría estar dado más bien por fuga del AL[37]. Al no existir un límite anatómico tan evidente entre ambos y considerando que los estudios muchas veces emplean indistintamente los términos, se revisarán en conjunto.

Por sus características anatómicas, estos abordajes son ultrasonográficos $y$, aunque ocasionalmente se pueden distinguir algunas de las ramas del nervio femoral, esta es una técnica que se realiza mediante una inyección perivascular.

Se posiciona al paciente en supino, con la extremidad en discreta rotación externa, para explorar con un transductor lineal de alta frecuencia el tercio medio del muslo con el fin de identificar el vértice del triángulo femoral (Figuras 7A y 7B). Una vez identificado, se puede ascender 1-2 cm en el caso del bloqueo del TF (Figuras 8A y 8B), o continuar hacia distal y puncionar 1-2 cm cefálico al hiato aductor (punto donde la arteria femoral se profundiza para convertirse en poplítea) en el caso del bloqueo del CA (Figuras 9A y 9B). La técnica de punción es en plano desde lateral, con una aguja de 80-100 mm.

Los volúmenes reportados oscilan entre 10 y 30 $\mathrm{ml}$. En el caso de las inyecciones proximales, un estudio de búsqueda de dosis estima la DE50 de ropivacaína $0,5 \%$ a nivel del vértice del TF es de 10,4 ml[38], mientras que otro estima en $20 \mathrm{ml}$ el volumen necesario para llenar el canal hacia distal desde el TF, pero considerando que con $10 \mathrm{ml}$ o más, también hay difusión hacia proximal[39]. En el caso de inyecciones más distales, se ha estimado en estudios cadavéricos que $10 \mathrm{ml}$ permean hacia el plexo poplíteo posterior[40] y $15 \mathrm{ml}$ hacia ambos extremos del CA[41]. 


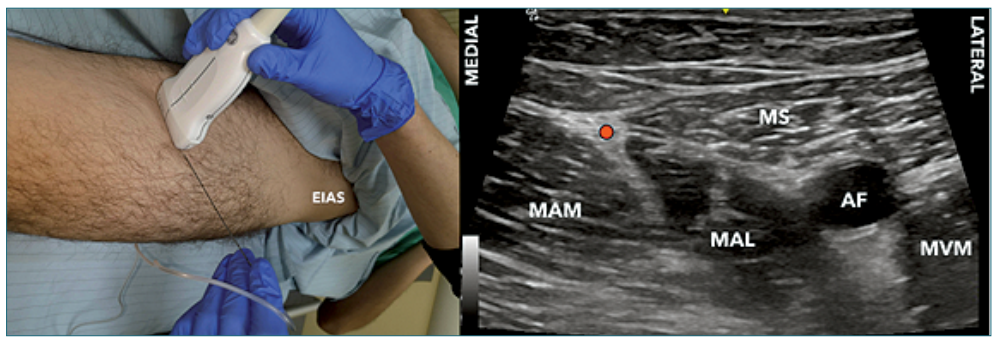

Figura 7. Vértice triángulo femoral (VTF). A. Posición transductor BTF; B. Sonoanatomía VTF: MVM: músculo vasto medial; MS: músculo sartorio; MAL: músculo aductor largo; AF: arteria femoral superficial; Punto azul: unión bordes mediales MAL y MS.

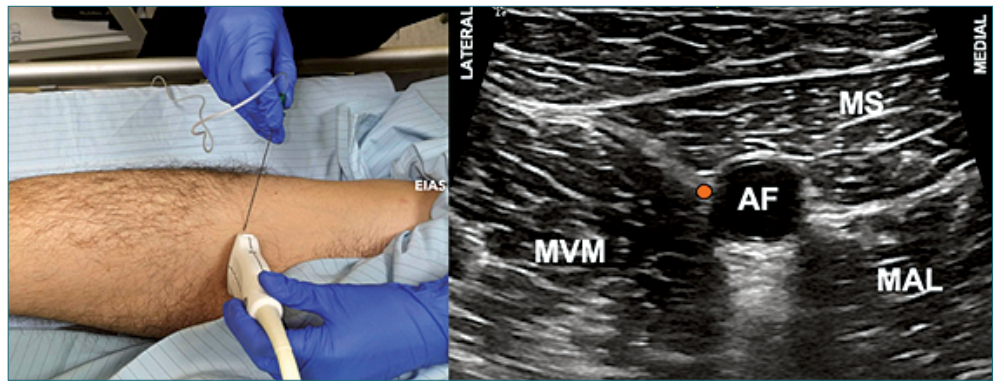

Figura 8. Bloqueo del triángulo femoral (BTF). A. Posición transductor BTF; B. Sonoanatomía BTF: MVM: músculo vasto medial; MS: músculo sartorio; MAL: músculo aductor largo; AF: arteria femoral superficial; Punto naranjo: sitio de inyección.

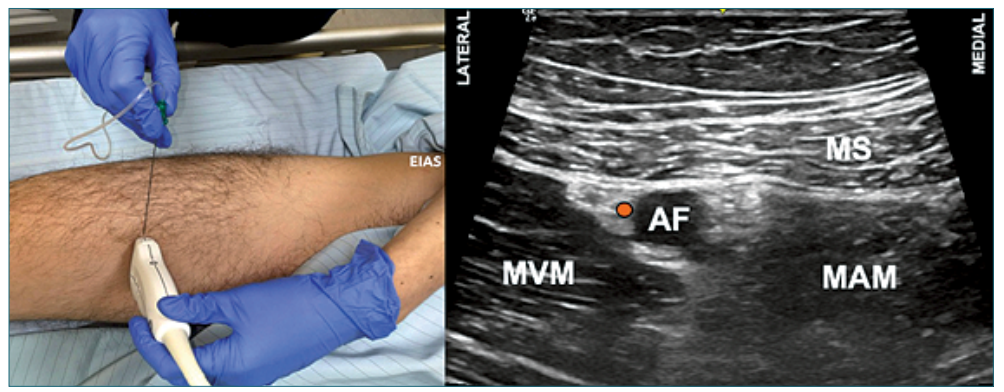

Figura 9. Bloqueo del canal aductor (BCA). A. Posición transductor BCA; B. Sonoanatomía BCA: MVM: músculo vasto medial; MS: músculo sartorio; MAM: músculo aductor magno; AF: arteria femoral superficial; Punto naranjo: sitio de inyección.

Se realiza mediante una inyección para administrar el $\mathrm{AL}$ en el aspecto lateral la arteria femoral superficial a nivel subsartorial en el caso del bloqueo del TF y bajo la MVA en el del bloqueo del CA.

Si bien hay poca evidencia, los catéteres instalados proximalmente (TF) tendrían un desempeño analgésico superior, con resultados similares desde el punto de vista de la funcionalidad, que los distales[42]-[45].

Como en toda técnica perivascular, existe el riego de lesión de los vasos femorales e inyección intravascular. Se describe además la posibilidad de pérdida de fuerza de cuádriceps tardía secundaria a mionecrosis, la cual sería reversible[46].

\section{Bloqueo del nervio safeno}

Si bien este nervio puede bloquearse en abordajes más proximales (en el CA), su búsqueda selectiva puede lograr ahorrar bloqueo muscular innecesario para cirugías distales con necesidad de proveer analgesia adecuada para cirugía compleja de tobillo y pie.

Históricamente, se han descrito variadas técnicas basadas en reparos anatómicos y NE, pero sus tasas de éxito son variables, por lo que el uso de US resulta preferible[2]. El bloqueo transartorial con US se basa en la búsqueda del plano anatómico donde se encuentra el nervio (ya que éste es difícilmente visible) tras su salida del canal aductor. El uso de NE concomitante no entrega ventajas adicionales[2].

Se escanea la cara antero-medial del muslo, para identificar el canal aductor y el hiato (Figura 10A). Distalmente de ese punto se genera un plano entre los músculos vasto medial por lateral y sartorio por medial, donde es habitual encontrar la arteria genicular descendente (Figura 10B), que acompaña al NS. Desde lateral se inserta una aguja de $50-100 \mathrm{~mm}$ para inyectar dentro de este plano. 


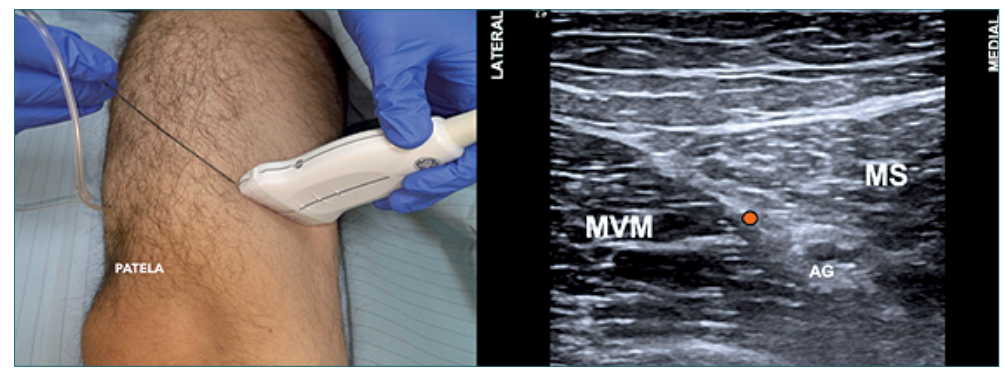

Figura 10. Bloqueo del nervio safeno (BNS). A. Posición transductor BNS; B. Sonoanatomía BNS: MVM: músculo vasto medial; MS: músculo sartorio; AG: arteria genicular; Punto naranjo: sitio de inyección.

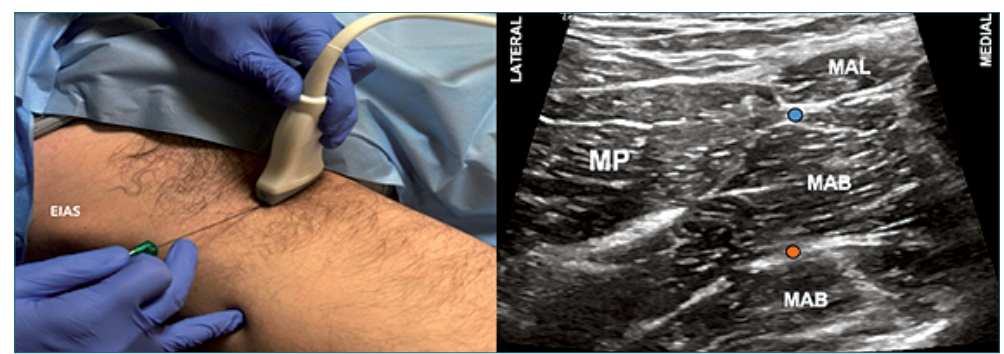

Figura 11. Bloqueo del nervio obturador distal (BNOD). A. Posición transductor BNO; B. Sonoanatomía BNO: MP: músculo pectíneo; MAL: músculo aductor largo; MAB: músculo aductor breve; MAM: músculo aductor magno; Punto naranjo: sitio de inyección.

No existen estudios de búsqueda de dosis para este abordaje. Habitualmente se emplean $10 \mathrm{ml}$ y al no ser común ver el nervio o la arteria, el punto de inyección es únicamente en el plano intermuscular.

De ser necesaria una técnica continua, dado que es un bloqueo superficial y en una zona de alta movilidad, es preferible un abordaje más proximal (bloqueo del (A).

Es considerada una técnica simple y segura.

\section{Bloqueo del nervio obturador}

Dado que abordajes únicos inguinales (Bloqueo 3:1 o fascia ilíaca) fallan en abarcar de manera consistente el NO, cuando el bloqueo de éste es necesario se debe pensar en un bloqueo del compartimento del psoas o uno específico de este nervio a nivel inguinal. Para evaluar el éxito debe considerarse que el clásico territorio cutáneo medial del muslo atribuido a la división anterior es altamente variable y muchas veces inexistente[47] y que la evaluación de la potencia muscular como alternativa para medir eficacia también es cuestionable ya que los NF y NC también aportan en la inervación aductora[48]. A esto se suma que la evidencia actual es imprecisa en aclarar el real aporte analgésico de este nervio en la práctica habitual de la cirugía de extremidad inferior[49],[50].

Las técnicas con NE han perdido popularidad debido a la variabilidad anatómica del nervio, su dificultad técnica y potenciales complicaciones. Por su parte, con US se puede abordar el nervio cuando se encuentra en su tronco común bajo la rama púbica in- ferior o una vez dividido entre los músculos aductores en el muslo. El uso de NE concomitante no genera un aporte técnico[2].

Para cualquiera de los dos niveles de bloqueo, se emplea un transductor lineal de alta frecuencia y el mapeo inicial es similar que el del bloqueo femoral, salvo que una vez identificados los vasos femorales la exploración continúa hacia medial con el fin de identificar los planos musculares de los aductores (Figura 11A); aquí se puede hacer tilt hacia cefálico para encontrar el plano entre los músculos pectíneo y obturador externo (bloqueo proximal)[51] o bien se puede realizar el bloqueo inyectando en ambos planos intermusculares para cubrir la rama anterior y posterior (bloqueo distal) (Figuras 11A y 11B).

No existen estudios de búsqueda de dosis para estos bloqueos. El volumen habitualmente empleado oscila entre 10 y $15 \mathrm{ml}$.

Para ambos bloqueos se describen punciones en plano desde lateral y fuera de plano con inclinación cefálica. Uno o dos sitios de depósito del AL serán necesarios según si la inyección es proximal o distal. Los bloqueos continuos están raramente indicados.

Al inyectar con inclinación cefálica existe el riesgo de punción intrapélvica.

\section{Bloqueo del nervio cutáneo femoral lateral}

Muchas veces alcanzado en los abordajes inguinales que buscan bloquear el NF, sin embargo, dado que sólo inerva piel en la cara antero-lateral del muslo, su relevancia clínica es cuestionable en cirugía de extre- 
midad inferior.

Desde el punto de vista técnico, es un bloqueo que en manos expertas es posible bloquear sólo con reparos anatómicos $\mathrm{O} \mathrm{NE}$, sin embargo, si se desea lograr consistencia dada la variabilidad anatómica, es mejor emplear una alternativa ultrasonográfica que permitirá además disminuir el número de pases de aguja. Para ello existen varias técnicas (infiltración dorsal al LI, perineural sobre el MS o perineural entre el MS y tensor de la fascia lata) no habiendo suficiente evidencia para determinar superioridad de una sobre otra[7],[52].

Para esto, con el paciente en supino, se realiza un mapeo transverso en la zona infrainguinal caudal a la EIAS (Figura 12A). El objetivo puede ser la imagen del nervio posado sobre el MS o el surco entre el músculo sartorio y el tensor de la fascia lata donde se forma un túnel aplanado de grasa rodeado por fascia lata en el cual está contenido el nervio (Figura 12B). El nervio o su compartimento se encontrará sólo bajo la fascia lata a este nivel.

No existen estudios de búsqueda de dosis para estos bloqueos. El volumen habitualmente empleado oscila entre 5 y $10 \mathrm{ml}$ y una vez lograda la imagen e identificado el espacio donde inyectar, se puede insertar una aguja de $50 \mathrm{~mm}$ tanto en plano desde lateral como fuera de plano. Bloqueos continuos estarían, al menos, raramente indicados.

Existe la posibilidad de difusión hacia el NF con el consiguiente riesgo de bloqueo motor del cuádriceps.

\section{Bloqueos del plexo sacro}

Del PS se originan solo tres nervios que inervan la extremidad inferior y que se deben considerar desde un punto de vista clínico. El nervio cutáneo femoral posterior del muslo, que rara vez requiere ser bloqueado, y los nervios tibial y peroneo que en la gran mayoría de los individuos viajan juntos hasta separarse en la fosa poplítea, constituyendo el nervio ciático.
En la era previa al ultrasonido existía una serie secuencial de abordajes desde proximal a distal (p.ej. parasacro, transglúteo, subglúteo, anterior medio femoral, poplíteo, etc.) que buscaban bloquear ya sea el plexo completo (acceso parasacro) o el NC. En la actualidad, si bien se emplean distintos abordajes, éstos quizás se han simplificado, donde lo relevante es la visualización de la estructura más allá del nivel mismo de punción.

\section{Bloqueo parasacro}

El bloqueo a este nivel tiene la virtud de cubrir las ramas articulares posteriores a la cadera y de bloquear en simultáneo el nervio cutáneo femoral posterior. Este abordaje se basa en la búsqueda del plexo a medida que se aleja de la línea media y se asoma por la escotadura ciática mayor, justo por anterior al músculo piriforme y profundo a la fascia presacra[53].

Este abordaje fue por mucho tiempo sólo realizado con $\mathrm{NE}$, sin embargo, en la última década se han descrito varias técnicas con US[54],[55]. Dadas las características de la zona de trabajo, este constituye un bloqueo profundo y técnicamente avanzado. Demanda experiencia en la interpretación de la imagen y muchas veces aún requiere apoyo de NE confirmatoria.

Con paciente en decúbito lateral o posición de sims se escanea sobre el hueso iliaco hacia ínferomedial con un transductor convexo buscando la pérdida de continuidad de este reparo óseo. Con el transductor orientado en el sentido transversal a nivel del borde superior del pliegue interglúteo y aproximadamente $8 \mathrm{~cm}$ hacia lateral de este reparo (Figura $13 \mathrm{~A})$, se escanea la zona buscando identificar, entre las sombras acústicas del sacro e isquion (escotadura ciática mayor) el músculo glúteo máximo y piriforme $y$, profundo a éstos los elementos del plexo, como un grupo de estructuras hiperecogénicas (Figura 13B) [54].

No hay estudios de volumen ni concentración para este abordaje. La punción se realiza en plano

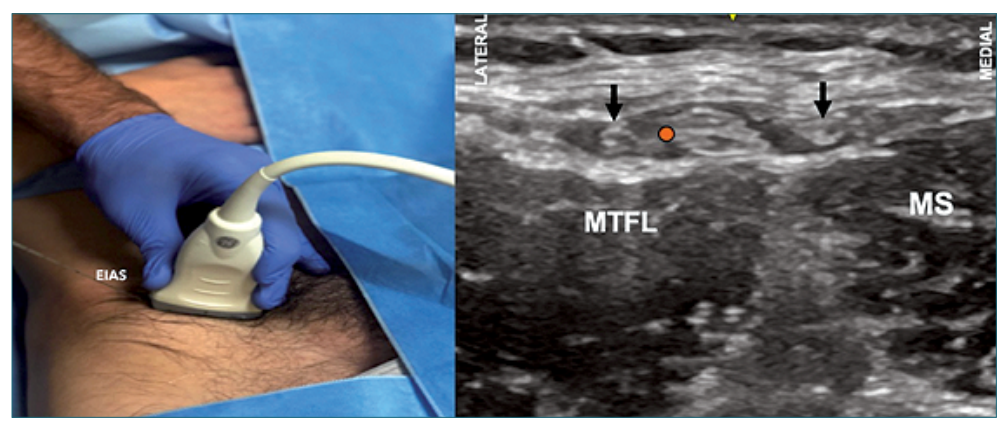

Figura 12. Bloqueo del nervio cutáneo femoral lateral (BNCFL). A. Posición transductor BNCFL; B. Sonoanatomía BNCFL: MS: músculo sartorio; MTFL: músculo tensor de la fascia lata; Punto naranjo: sitio de inyección. 

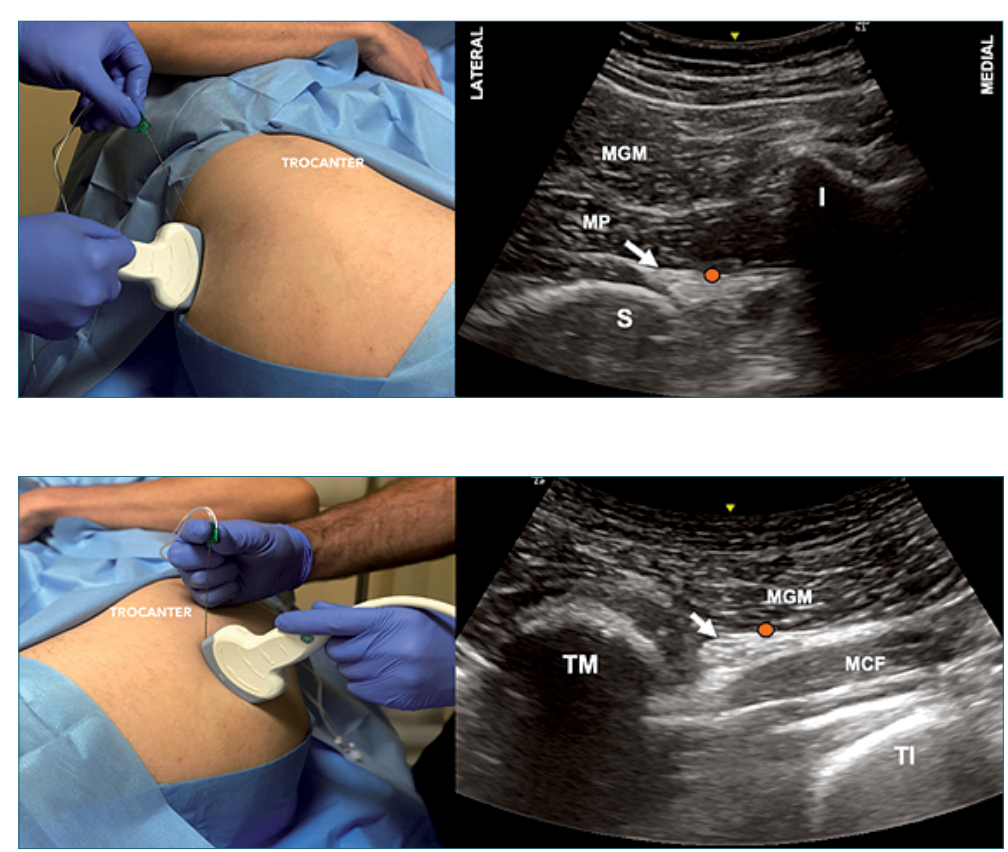

Figura 13. Bloqueo parasacro (BPS). A. Posición transductor BPS; B. Sonoanatomía BPS: I: isquion; S: sacro MGM: músculo glúteo mayor; MP: músculo piriformis; Punto naranjo: sitio de inyección.

Figura 14. Bloqueo del nervio ciático subglúteo (BNCSG). A. Posición transductor BNCSG; B. Sonoanatomía BNCSG. TI: tuberosidad isquiática; TM: trocánter mayor; MGM: músculo glúteo mayor; MCF: músculo cuadrado femoral; Punto naranjo: sitio de inyección.

desde lateral o fuera de plano con una aguja de 80$100 \mathrm{~mm}$ para inyectar $20 \mathrm{ml}$.

Si bien es factible la instalación de catéteres, su necesidad resulta más bien anecdótica, sobre todo considerando que existen abordajes más seguros[56].

La arteria glútea inferior se encuentra en las inmediaciones de este mismo plano, por lo que debe tenerse especial precaución durante la inyección y, por ser un bloqueo profundo, también requiere de las mismas precauciones que en el neuroeje desde el punto de vista de la hemostasia. Además, esta es una zona crítica desde el punto de vista de la vascularización del NC, por lo que se desaconseja el uso de vasoconstrictor para no correr el riesgo de comprometer la perfusión neural[57].

\section{Bloqueo del nervio ciático a nivel subglúteo}

El término subglúteo con la técnica de NE se refiere a una punción caudal al pliegue glúteo, sin embargo, con US se emplea más bien para referirse al lugar donde se deposita el $A L$, es decir, profundo (anterior) al músculo glúteo máximo.

El uso de NE fue la modalidad más popular hasta el inicio de la presente década, esto por su alta tasa de éxito y relativa facilidad técnica. El procedimiento original, realizado mediante una punción caudal al punto medio de una línea que une la tuberosidad isquiática y el trocánter mayor, fue reemplazado por una aproximación más medial, en posición prona, que ha demostrado mayor éxito y menores tiempos de inicio de bloqueo[58],[59]. Al neuroestimular, la respuesta objetivo debería ser la flexión plantar/inversión del pie, que comparada a la dorsiflexión/eversión (nervio peroneo), presenta mayor porcentaje de éxito y menor tiempo a bloqueo sensitivo y motor[60]. La doble inyección comparada a inyección única produce un bloqueo de inicio más rápido sin diferencias en la efectividad de la técnica[61]. El uso de US permite disminuir la dosis de AL empleado y la inyección circunferencial aumenta el bloqueo completo[2]. No hay evidencia que avale el uso rutinario de NE concomitante si el nervio se visualiza bien[2].

Para la técnica con US de este bloqueo, se ubica al paciente en decúbito lateral o prono, para luego explorar con un transductor curvo de baja frecuencia la región entre el trocánter mayor y la tuberosidad isquiática (Figura 14A). El nervio se observa como una imagen fascicular y triangular ventral al glúteo máximo y dorsal al cuadrado femoral, en el compartimento subglúteo (Figura 14B).

Existe un estudio de búsqueda de dosis que estableció en $14 \mathrm{~mL}$ el VEM95 de mepivacaína 1,5\% la técnica con US mientras que de 29 mL con NE[62].

La punción normalmente se realiza en plano desde lateral a medial, utilizando una aguja de 80 a $100 \mathrm{~mm}$, buscando rodear el nervio ciático o bien depositar la solución en el plano entre los músculos descritos, ambos con similar efectividad[63]. Si bien 
la inyección subparaneural a este nivel es factible, es técnicamente mucho más demandante.

Al comparar los bloqueos continuos con catéteres estimulantes realizados con a este nivel versus poplíteo, se observa una disminución de requerimientos de $\mathrm{AL}$ con analgesia comparable[64].

\section{Bloqueo del nervio ciático a nivel poplíteo}

Constituye el abordaje más frecuente del NC, ya sea por vía lateral o posterior. El tejido conectivo extraneural de esta zona, denominado paraneuro, cobra especial relevancia ya que ha establecido distintas técnicas para el procedimiento[65],[66].

Las técnicas guiadas por NE, ya sea por vía lateral o posterior, si bien resultan ser técnicamente factibles[67], han sido consistentemente reemplazadas por el uso de US, en base a la mayor tasa de éxito y menores requerimientos de $\mathrm{AL}[68],[69]$.

Para su realización se posiciona al paciente en supino con la pierna elevada de manera tal de poder ubicar el transductor bajo el muslo, o también se puede poner en prono o decúbito lateral (Figura 15A). Con un transductor lineal de alta frecuencia se escanea la fosa poplítea donde se pueden identificar los vasos homónimos y el nervio ciático (o sus componentes) superficial y lateral a ellos (Figura 15B). El nervio presenta anisotropía significativa debido a su disposición anatómica, por lo que angular el transductor permite la optimización de la imagen. La punción se puede realizar tanto en plano como fuera de plano con una aguja de entre 50 y 100 mm según la técnica de punción.

La evidencia actual presenta considerable heterogeneidad en las metodologías utilizadas, mediante el método secuencial de Dixon con NE el VEM50 de mepivacaína 1,5\% fue de $20 \pm 3$ ml[70], mientras que con US el VEM50 y 90 de ropivacaína 0,75\% tras una inyección subparaneural es de 6,14 y 8,9 ml, respectivamente[71]. Por otra parte, al buscar dirigidamente el VEM90 de lidocaína 1\%-bupivacaína 0,25\% subparaneural resulta en 13,3 ml (Cl 95\% 10,2-16,4 $\mathrm{ml})[72]$.
Lo recomendable es abordar el NC cercano al límite proximal de la fosa poplítea, inmediatamente previo o a nivel de su bifurcación, inyectando el $\mathrm{AL}$ a nivel subparaneural, logrando así la clara división de ambos nervios dentro de esta capa de tejido conectivo extraneural. Esta técnica, comparada a depositar $\mathrm{AL}$ externamente, demuestra mayor éxito y menores tiempos a inicio de bloqueo sensitivo-motor[65],[66].

El uso de catéteres estimulantes permite disminuir la masa de droga requerida, sin objetivarse diferencia en control de dolor[73],[74]. Las recomendaciones actuales sugieren que el posicionamiento de la punta del catéter a nivel subparaneural comparado a extraparaneural tiene una tasa de éxito mayor y a la vez, el instalarlo en eje largo presentaría un mejor perfil analgésico con la ventaja además de menor riesgo de desplazamiento del catéter[75],[76].

El acceso al espacio subparaneural requiere de especial precaución. Una vez dentro, al inyectar debiesen delimitarse ambos componentes del NC, sin evidenciarse deformación de ellos.

\section{Bloqueo IPACK}

El bloqueo del interespacio entre la arteria poplítea y la cápsula posterior de la rodilla, corresponde a una alternativa analgésica que busca bloquear aferencias ciáticas y posiblemente del NO en el contexto de cirugía de rodilla[77]. En ese sentido, su objetivo es complementar el bloqueo del componente anterior, pero evitando el compromiso motor de pierna y el enmascaramiento de una potencial lesión quirúrgica del NP, lo anterior en el contexto de un bloqueo ciático clásico[78]. Así, tras la bifurcación del NC y por encima del pliegue poplíteo, el NT emite ramos hacia la rodilla, los que van a conformar el plexo poplíteo posterior e inervar los meniscos, la cápsula posterior de la articulación, los ligamentos cruzados y la almohadilla de grasa infrapatelar. Estudios anatómicos muestran que la rama posterior del NO también podría ser alcanzada en un bloqueo IPACK[77].

Constituye es un abordaje eminentemente ultrasonográfico donde se posiciona un transductor curvo

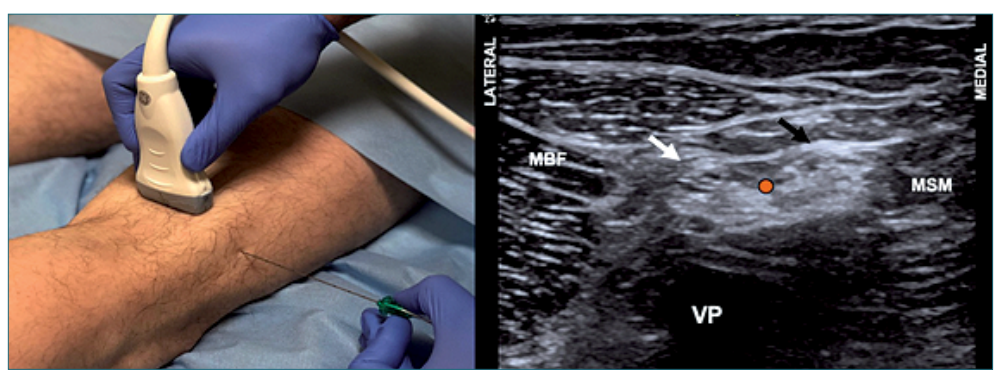

Figura 15. Bloqueo del nervio ciático poplíteo (BNCP). A. Posición transductor BNCP; B. Sonoanatomía BNCP. VP: vasos poplíteos; MBF: músculo bíceps femoral; MSM: músculo semimembranoso; Flecha blanca: componente peroneo; Flecha negra: componente tibial; Punto naranjo: sitio de inyección subparaneural. 


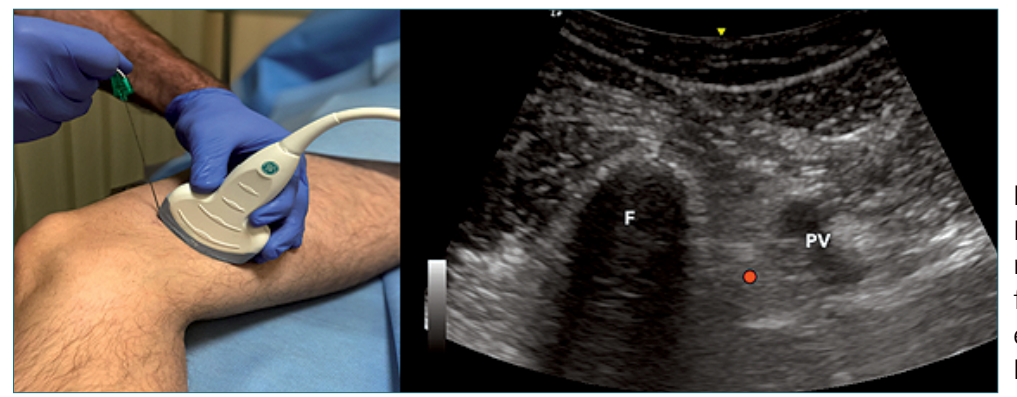

Figura 16. Bloqueo IPACK (IPACKB).A. Posición transductor IPACKB; B. Sonoanatomía IPACKB. VP: vasos poplíteos; F: fémur; Punto naranjo: sitio de Inyección entre Popliteal Artery y Capsule of the Knee.

de baja frecuencia en la cara medial del muslo a nivel del pliegue poplíteo (Figura 16A), ubicando la sombra acústica producida por el fémur hacia anterior y la arteria poplítea hacia dorsal (Figura 16B)[78]. Alternativamente se describe una técnica distal muy similar, donde en vez de buscar el cuerpo del fémur, se sitúa el transductor a nivel de los cóndilos femorales[77]. Con el transductor situado en eje corto en relación con arteria poplítea y fémur, se punciona en la cara medial del muslo distal en una orientación de anteromedial a pósterolateral, buscando infiltrar el espacio existente entre la arteria y fémur de manera de abar- car la zona desde lateral a medial, inyectando en la medida que la aguja se retira.

No existen estudios de dosis. La descripción original utilizó $30 \mathrm{~mL}$ de ropivacaína al 0,2\%, lo cual ha sido repetido en comunicaciones posteriores[79].

El sitio objetivo en donde depositar la solución de AL es el espacio ubicado entre la arteria poplítea y la cápsula posterior de la rodilla. No se ha descrito el uso de catéteres para este abordaje.

Se describe la posibilidad de lesión del NS secundario al sitio de punción.

\section{Referencias}

1. Farny J, Drolet P, Girard M. Anatomy of the posterior approach to the lumbar plexus block. Can J Anaesth. 1994 Jun;41(6):4805. https://doi.org/10.1007/ BF03011541 PMID:8069987

2. Tran DQ, Salinas FV, Benzon HT, Neal JM. Lower extremity regional anesthesia: essentials of our current understanding. Reg Anesth Pain Med. 2019 Jan;44(2):143143-80. https:// doi.org/10.1136/rapm-2018000019 PMID:30635506

3. Salinas FV. Lower ExtremityBlocks of the Lumbar Plexus and Lumbar Plexus Peripheral Nerves. Fifth edit. Neal JM, Tran DQH, Salinas FV., editors. A Practical Approach to Regional Anesthesiology and Acute Pain Medicine. LWW; 2017.

4. Capdevila $X$, Macaire $P$, Dadure $C$, Choquet $\mathrm{O}$, Biboulet $\mathrm{P}$,
Ryckwaert $Y$, et al. Continuous psoas compartment block for postoperative analgesia after total hip arthroplasty: new landmarks, technical guidelines, and clinical evaluation. Anesth Analg. 2002 Jun;94(6):1606-13. https:// doi.org/10.1213/00000539200206000-00045

PMID:12032037

5. Ilfeld BM, Loland VJ, Mariano ER. Prepuncture ultrasound imaging to predict transverse process and lumbar plexus depth for psoas compartment block and perineural catheter insertion: a prospective, observational study. Anesth Analg. 2010 Jun;110(6):17258. https://doi.org/10.1213/ ANE.0b013e3181db7ad3 PMID:20385611

6. Tomaszewski KA, Popieluszko P, Henry BM, Roy J, Sanna B, Kijek $M R$, et al. The surgical anatomy of the lateral femoral cutaneous nerve in the inguinal region: a meta-analysis. Hernia. 2016 Oct;20(5):649-57. https://doi. org/10.1007/s10029-016-14937 PMID:27115766

7. Nielsen TD, Moriggl B, Barckman J, Kølsen-Petersen JA, Søballe K, Børglum J, et al. The Lateral Femoral Cutaneous Nerve: Description of the Sensory Territory and a Novel Ultrasound-Guided Nerve Block Technique. Reg Anesth Pain Med. 2018 May;43(4):35766. https://doi.org/10.1097/ AAP.0000000000000737 PMID:29381568

8. Vloka JD, Hadzic A, Drobnik L, Ernest A, Reiss W, Thys DM. Anatomical Landmarks for Femoral Nerve Block: A Comparison of Four Needle Insertion Sites. Anesth Analg [Internet]. 1999 Dec;89(6):1467. Available from: http://insights.ovid. com/crossref $n=00000539$ - 


\section{0-00028}

9. Kennedy JC, Alexander IJ, Hayes KC. Nerve supply of the human knee and its functional importance. Am J Sports Med. 1982 Nov-Dec;10(6):329-35. https://7/036354658201000601 PMID:6897495

10. Gardner $\mathrm{E}$. The innervation of the knee joint [Internet]. Anat Rec. 1948 May;101(1):109-30. Available from: http://doi.wiley. com/10.1002/ar.1091010111 https://doi.org/10.1002/ ar.1091010111 PMID:18915634

11. Short AJ, Barnett JJ, Gofeld M, Baig E, Lam K, Agur AM, et al. Anatomic Study of Innervation of the Anterior Hip Capsule: Implication for Image-Guided Intervention. Reg Anesth Pain Med. 2018 Feb;43(2):186-92. PMID:29140962

12. Sunderland S, Ray LJ. The intraneural topography of the sciatic nerve and its popliteal divisions in man [Internet]. Brain. 1948 Sep;71(Pt. 3):242-73. Available from: https://academic. oup.com/brain/article-lookup/ doi/10.1093/brain/71.3.242 https://doi.org/10.1093/brain/71.3.242 PMID:18099549

13. Bendtsen TF, Moriggl B, Chan V, Børglum J. The Optimal Analgesic Block for Total Knee Arthroplasty. Reg Anesth Pain Med. 2016 Nov/Dec;41(6):7119. https://doi.org/10.1097/ AAP.0000000000000485 PMID:27685346

14. Gardner $E$. The innervation of the hip joint [Internet]. Anat Rec. 1948 Jul;101(3):353-71. Available from: http://doi.wiley. com/10.1002/ar.1091010111 https://doi.org/10.1002/ ar.1091010309 PMID:18873153

15. Gerhardt $M$, Johnson $K$, Atkinson $R$, Snow B, Shaw C, Brown $A$, et al. Characterisation and classification of the neural anatomy in the human hip joint. Hip
Int. 2012 Jan-Feb;22(1):75-81.

https://doi.org/10.5301/

HIP.2012.9042 PMID:22344482

16. Orduña Valls JM, Vallejo $R$, López Pais $P$, Soto E, Torres Rodríguez D, Cedeño DL, et al. Anatomic and ultrasonographic evaluation of the knee sensory innervation a cadaveric study to determine anatomic targets in the treatment of chronic knee pain. Reg Anesth Pain Med. 2017 Jan/Feb;42(1):908. https://doi.org/10.1097/ AAP.0000000000000516 PMID:27922951

17. Karmakar MK, Ho AM, Li X, Kwok WH, Tsang K, Ngan Kee WD. Ultrasound-guided lumbar plexus block through the acoustic window of the lumbar ultrasound trident. Br J Anaesth. 2008 Apr;100(4):533-7. https:// doi.org/10.1093/bja/aen026 PMID:18344573

18. Karmakar MK, Li JW, Kwok WH, Hadzic A. Ultrasoundguided lumbar plexus block using a transverse scan through the lumbar intertransverse space: a prospective case series. Reg Anesth Pain Med. 2015 Jan-Feb;40(1):75-81. https://doi.org/10.1097/ AAP.0000000000000168 PMID:25469756

19. Sauter $A R$, Ullensvang $K$, Bendtsen TF, Borglum J. Shamrock Method: a new and promising techniquefor ultrasound-guided lumbar plexus block. Br J Anaesth. 2013;111 eLetters:533-7. https://doi. org/10.1093/bja/el_9814.

20. Lin JA, Lu HT. Solution to the challenging part of the Shamrock method during lumbar plexus block [Internet]. $\mathrm{Br}$ J Anaesth. 2014 Sep;113(3):5167. Available from: https://linkinghub.elsevier.com/retrieve/ pii/S0007091217317786 https:// doi.org/10.1093/bja/aeu277
PMID:25135892

21. Arnuntasupakul V, Chalachewa $T$, Leurcharusmee $P$, Tiyaprasertkul W, Finlayson RJ, Tran DQ. Ultrasound with neurostimulation compared with ultrasound guidance alone for lumbar plexus block. Eur J Anaesthesiol [Internet]. 2017 Nov; 1. https://doi.org/10.1097/ EJA.0000000000000736.

22. Sauter $A R$, Ullensvang $K$, Niemi G, Lorentzen HT, Bendtsen TF, Børglum J, et al. The Shamrock lumbar plexus block: A dosefinding study. Eur J Anaesthesiol. 2015 Nov;32(11):764-70. https://doi.org/10.1097/ EJA.0000000000000265 PMID:26426575

23. Horlocker TT, Wedel DJ, Rowlingson JC, Enneking FK, Kopp SL, Benzon HT, et al. Regional anesthesia in the patient receiving antithrombotic or thrombolytic therapy: American Society of Regional Anesthesia and Pain Medicine EvidenceBased Guidelines (Third Edition) [Internet]. Reg Anesth Pain Med. 2010 Jan-Feb;35(1):64-101. Available from: http://content. wkhealth.com/linkback/open url?sid=WKPTLP:landingpage $\& n=00115550-201001000-0$ 0013 https://doi.org/10.1097/ AAP.0b013e3181c15c70 PMID:20052816

24. Gadsden JC, Lindenmuth DM, Hadzic A, Xu D, Somasundarum L, Flisinski KA. Lumbar plexus block using high-pressure injection leads to contralateral and epidural spread. Anesthesiology. 2008 Oct;109(4):6838. https://doi.org/10.1097/ ALN.0b013e31818631a7 PMID:18813048

25. Capdevila $X$, Biboulet $P$, Bouregba $M$, Barthelet $Y$, Rubenovitch J, d'Athis F. Comparison of the three-in-one and fascia iliaca compartment blocks in 
adults: clinical and radiographic analysis. Anesth Analg. 1998 May;86(5):1039-44. https:// doi.org/10.1213/00000539199805000-00025 PMID:9585293

26. Marhofer $P$, Našel $C$, Sitzwohl C, Kapral S. Magnetic resonance imaging of the distribution of local anesthetic during the threein-one block. Anesth Analg. 2000 Jan;90(1):119-24. https:// doi.org/10.1097/00000539200001000-00027

PMID:10624991

27. Swenson JD, Davis JJ, Stream JO, Crim JR, Burks RT, Greis PE. Local anesthetic injection deep to the fascia iliaca at the level of the inguinal ligament: the pattern of distribution and effects on the obturator nerve [Internet]. J Clin Anesth. 2015 Dec;27(8):6527. https://doi.org/10.1016/j. jclinane.2015.07.001 PMID:26277873

28. Dalens B, Vanneuville G, Tanguy $A$. Comparison of the fascia iliaca compartment block with the 3-in-1 block in children. Anesth Analg. 1989 Dec;69(6):705-13. https:// doi.org/10.1213/00000539198912000-00003 PMID:2589650

29. Stevens M, Harrison G, McGrail M. A modified fascia iliaca compartment block has significant morphine-sparing effect after total hip arthroplasty. Anaesth Intensive Care. 2007 Dec;35(6):949-52. https://doi.g/ 10.1177/0310057X0703500615 PMID:18084988

30. Hebbard P, Ivanusic J, Sha S. Ultrasound-guided suprainguinal fascia iliaca block: a cadaveric evaluation of a novel approach. Anaesthesia. 2011 Apr;66(4):300-5. https://doi.org/10.1111/ j.1365-2044.2011.06628.x

\section{PMID:21401544}

31. Vermeylen $K$, Soetens $F$, Leunen I, Hadzic A, Van Boxtael S, Pomés J, et al. The effect of the volume of supra-inguinal injected solution on the spread of the injectate under the fascia iliaca: a preliminary study [Internet]. J Anesth. 2018 Dec;32(6):908-13. https://doi.org/10.1007/s00540018-2558-9 PMID:30250982

32. Vermeylen $K$, Desmet $M$, Leunen I, Soetens F, Neyrinck $A$, Carens $D$, et al. Supra-inguinal injection for fascia iliaca compartment block results in more consistent spread towards the lumbar plexus than an infrainguinal injection: A volunteer study. Reg Anesth Pain Med. 2019;44(4):483-91. https:// doi.org/10.1136/rapm-2018100092.

33. Desmet M, Vermeylen $\mathrm{K}$, Van Herreweghe I, Carlier L, Soetens F, Lambrecht S, et al. A longitudinal supra-inguinal fascia iliaca compartment block reduces morphine consumption after total hip arthroplasty. Reg Anesth Pain Med. 2017 May/Jun;42(3):32733. https://doi.org/10.1097/ AAP.0000000000000543 PMID:28059869

34. Girón-Arango L, Peng PW, Chin KJ, Brull R, Perlas A. Pericapsular Nerve Group (PENG) Block for Hip Fracture. Reg Anesth Pain Med. 2018 Nov;43(8):85963. https://doi.org/10.1097/ AAP.0000000000000847 PMID:30063657

35. Casati A, Baciarello M, Di Cianni S, Danelli G, De Marco G, Leone $S$, et al. Effects of ultrasound guidance on the minimum effective anaesthetic volume required to block the femoral nerve. $\mathrm{Br} J$ Anaesth. 2007 Jun;98(6):8237. https://doi.org/10.1093/bja/ aem100 PMID:17478453

36. Anns JP, Chen EW, Nirkavan
N, McCartney CJ, Awad IT. A comparison of sartorius versus quadriceps stimulation for femoral nerve block: a prospective randomized doubleblind controlled trial. Anesth Analg. 2011 Mar;112(3):72531. https://doi.org/10.1213/ ANE.0b013e3182052213 PMID:21127273

37. Wong WY, Bjørn S, Strid JM, Børglum J, Bendtsen TF. Defining the Location of the Adductor Canal Using Ultrasound. Reg Anesth Pain Med. 2017 Mar/Apr;42(2):241-5. https://doi.org/10.1097/ AAP.0000000000000539 PMID:28002228

38. Tao $Y$, Zheng SQ, Xu T, Wang G, Wang Y, Wu AS, et al. Median effective volume of ropivacaine $0.5 \%$ for ultrasound-guided adductor canal block. J Int Med Res. 2018 Oct;46(10):4207-13. https://doi. rg/10.1177/0300060518791685 PMID:30124351

39. Jæger $P$, Jenstrup MT, Lund J, Siersma $V$, Brøndum V, Hilsted $\mathrm{KL}$, et al. Optimal volume of local anaesthetic for adductor canal block: using the continual reassessment method to estimate ED95. Br J Anaesth. 2015 Dec;115(6):920-6. https:// doi.org/10.1093/bja/aev362 PMID:26582853

40. Runge C, Moriggl B, Børglum J, Bendtsen TF. The Spread of Ultrasound-Guided Injectate From the Adductor Canal to the Genicular Branch of the Posterior Obturator Nerve and the Popliteal Plexus: A Cadaveric Study. Reg Anesth Pain Med. 2017 Nov/Dec;42(6):72530. https://doi.org/10.1097/ AAP.0000000000000675 PMID:28937534

41. Andersen $\mathrm{HL}$, Andersen SL, Tranum-Jensen J. The spread of injectate during saphenous ner- 
ve block at the adductor canal: a cadaver study. Acta Anaesthesiol Scand. 2015 Feb;59(2):23845. https://doi.org/10.1111/ aas. 12451 PMID:25496028

42. Mariano ER, Kim TE, Wagner MJ, Funck N, Harrison TK, Walters $\mathrm{T}$, et al. A randomized comparison of proximal and distal ultrasound-guided adductor canal catheter insertion sites for knee arthroplasty. J Ultrasound Med. 2014 Sep;33(9):165362. https://doi.org/10.7863/ ultra.33.9.1653 PMID:25154949

43. Chuan A, Lansdown A, Brick KL, Bourgeois AJ, Pencheva LB, Hue $B$, et al.; Continuous Catheters in Adductor Canal versus Femoral Triangle (The CAFE study) investigators. Adductor canal versus femoral triangle anatomical locations for continuous catheter analgesia after total knee arthroplasty: a multicentre randomised controlled study [Internet]. Br J Anaesth. 2019 Sep;123(3):360-7. https://doi. org/10.1016/j.bja.2019.03.021 PMID:31056239

44. Sztain JF, Machi AT, Kormylo NJ, Abramson WB, Madison SJ, Monahan AM, et al. Continuous Adductor Canal Versus Continuous Femoral Nerve Blocks: Relative Effects on Discharge Readiness Following Unicompartment Knee Arthroplasty. Reg Anesth Pain Med. 2015 Sep-Oct;40(5):559-67. https://doi.org/10.1097/ AAP.0000000000000279 PMID:26115189

45. Meier AW, Auyong DB, Yuan SC, Lin SE, Flaherty JM, Hanson NA. Comparison of Continuous Proximal Versus Distal Adductor Canal Blocks for Total Knee Arthroplasty: A Randomized, Double-Blind, Noninferiority Trial. Reg Anesth Pain Med. 2018 Jan;43(1):3642. https://doi.org/10.1097/

\section{AAP.0000000000000692 PMID:29140959}

46. Neal JM, Salinas FV, Choi DS. Local Anesthetic-Induced Myotoxicity After Continuous Adductor Canal Block. Reg Anesth Pain Med. 2016 Nov/Dec;41(6):723-

7. https://doi.org/10.1097/ AAP.0000000000000466 PMID:27662067

47. Bouaziz $H$, Vial F, Jochum $D$, Macalou D, Heck M, Meuret $P$, et al. An evaluation of the cutaneous distribution after obturator nerve block. Anesth Analg. 2002 Feb;94(2):445-9. PMID:11812716

48. Jochum $D$, lohom $G$, Choquet O, Macalou D, Ouologuem S, Meuret $P$, et al. Adding a selective obturator nerve block to the parasacral sciatic nerve block: an evaluation. Anesth Analg. 2004 Nov;99(5):15449. https://doi.org/10.1213/01. ANE.0000136464.86801.EE PMID:15502062

49. Nielsen ND, Runge C, Clemmesen L, Børglum J, Mikkelsen LR, Larsen JR, et al. An Obturator Nerve Block does not Alleviate Postoperative Pain after Total Hip Arthroplasty: a Randomized Clinical Trial. Reg Anesth Pain Med. 2019 Jan;44(4):466-71. https://doi.org/10.1136/rapm2018-100104 PMID:30679337

50. Runge $C$, Børglum J, Jensen JM, Kobborg T, Pedersen A, Sandberg J, et al. The analgesic effect of obturator nerve block added to a femoral triangle block after total knee arthroplasty a randomized controlled trial. Reg Anesth Pain Med. 2016 Jul-Aug;41(4):445-51. https://doi.org/10.1097/ AAP.0000000000000406 PMID:27171822

51. Taha AM. UltrasoundGuided Obturator Nerve Block. Anesth Analg [Internet]. 2012 Jan;114(1):236-
9. Available from: https:// insights.ovid.com/crossref an=00000539-20120100000034 https://doi.org/10.1213/ ANE.0b013e318237fb40.

52. Hara K, Sakura S, Shido A. Ultrasound-guided lateral femoral cutaneous nerve block: comparison of two techniques. Anaesth Intensive Care. 2011 Jan;39(1):69-72. https://doi.g/1 $0.1177 / 0310057 \times 1103900111$ PMID:21375093

53. Mansour NY. Reevaluating the sciatic nerve block: another landmark for consideration. Reg Anesth [Internet]. 18(5):322-3. Available from: http://www.ncbi. nlm.nih.gov/pubmed/8268124

54. Taha AM. A simple and successful sonographic technique to identify the sciatic nerve in the parasacral area. Can J Anaesth. 2012 Mar;59(3):263-7. https:// doi.org/10.1007/s12630-0119630-3 PMID:22139964

55. Ben-Ari AY, Joshi R, Uskova A, Chelly JE. Ultrasound localization of the sacral plexus using a parasacral approach. Anesth Analg. 2009 Jun;108(6):197780. https://doi.org/10.1213/ ane.0b013e3181a04d8e PMID:19448235

56. Gaertner $E$, Lascurain $P$, Venet $C$, Maschino $X$, Zamfir A, Lupescu R, et al. Continuous parasacral sciatic block: a radiographic study. Anesth Analg. 2004 Mar;98(3):831-4. https://doi.org/10.1213/01. ANE.0000099368.62200.01 PMID:14980947

57. Brull R, Hadzic A, Reina MA, Barrington MJ. Pathophysiology and Etiology of Nerve Injury Following Peripheral Nerve Blockade [Internet]. Reg Anesth Pain Med. 2015 SepOct;40(5):479-90. Available from: http://www.ncbi.nlm. nih.gov/pubmed/25974275 https://doi.org/10.1097/ 
AAP. 0000000000000125 PMID:25974275

58. di Benedetto P, Bertini L, Casati A, Borghi B, Albertin A, Fanelli $G$. A new posterior approach to the sciatic nerve block: a prospective, randomized comparison with the classic posterior approach. Anesth Analg. 2001 Oct;93(4):1040-4. https:// doi.org/10.1097/00000539200110000-00049 PMID:11574380

59. Wadhwa A, Tlucek H, Sessler D. A simple approach to the sciatic nerve that does not require geometric calculations or multiple landmarks. Anesth Analg. 2010 Mar;110(3):95863. https://doi.org/10.1213/ ANE.0b013e3181c95b4e PMID:20032026

60. Sukhani R, Nader A, Candido KD, Doty R Jr, Benzon $H T$, Yaghmour E, et al. Nerve stimulator-assisted evoked motor response predicts the latency and success of a singleinjection sciatic block. Anesth Analg. 2004 Aug;99(2):584-8. https://doi.org/10.1213/01. ANE.0000122823.50592.C9 PMID:15271744

61. Taboada M, Álvarez J, Cortés J, Rodríguez J, Atanassoff PG. Is a double-injection technique superior to a single injection in posterior subgluteal sciatic nerve block? Acta Anaesthesiol Scand. 2004 Aug;48(7):8837. https://doi.org/10.1111/ j.1399-6576.2004.00441.x PMID:15242434

62. Danelli G, Ghisi D, Fanelli A, Ortu A, Moschini E, Berti M, et al. The effects of ultrasound guidance and neurostimulation on the minimum effective anesthetic volume of mepivacaine $1.5 \%$ required to block the sciatic nerve using the subgluteal approach. Anesth Analg. 2009 Nov;109(5):1674-
8. https://doi.org/10.1213/

ANE.0b013e3181b92372 PMID:19843807

63. Salinas FV. Lower ExtremitySciatic Nerve Block. In: A Practical Approach to Regional Anesthesiology and Acute Pain Medicine. 2017.

64. Taboada M, Rodríguez J, Valiño C, Vazquez M, Laya A, Garea $M$, et al. A prospective, randomized comparison between the popliteal and subgluteal approaches for continuous sciatic nerve block with stimulating catheters. Anesth Analg. 2006 Jul;103(1):244-7. https://doi.org/10.1213/01. ane.0000220946.64570.73 PMID:16790661

65. Tran DQ, González AP, Bernucci F, Pham K, Finlayson RJ. A randomized comparison between bifurcation and prebifurcation subparaneural popliteal sciatic nerve blocks. Anesth Analg. 2013 May;116(5):11705. https://doi.org/10.1213/ ANE.0b013e31828a73be PMID:23492960

66. Perlas A, Wong P, Abdallah F, Hazrati LN, Tse C, Chan V. Ultrasound-guided popliteal block through a common paraneural sheath versus conventional injection: a prospective, randomized, double-blind study. Reg Anesth Pain Med. 2013 May-Jun;38(3):218-25. https://doi.org/10.1097/ AAP.0b013e31828db12f PMID:23558372

67. Hadzic A, Vloka JD. A Comparison of the Posterior versus Lateral Approaches to the Block of the Sciatic Nerve in the Popliteal Fossa. Anesthesiology [Internet]. 1998 Jun;88(6):1480-6. Available from: https://insights. ovid.com/crossref? 00000542199806000-00010 https:// doi.org/10.1097/00000542199806000-00010.
68. Perlas A, Brull R, Chan VW, McCartney CJ, Nuica A, Abbas $S$. Ultrasound guidance improves the success of sciatic nerve block at the popliteal fossa. Reg Anesth Pain Med. 2008 May-Jun;33(3):259-65. https:// doi.org/10.1097/00115550200805000-00011 PMID:18433678

69. van Geffen GJ, van den Broek E, Braak GJ, Giele JL, Gielen MJ, Scheffer GJ. A prospective randomised controlled trial of ultrasound guided versus nerve stimulation guided distal sciatic nerve block at the popliteal fossa. Anaesth Intensive Care. 2009 Jan;37(1):32-7. https://doi.g/10 $.1177 / 0310057 \times 0903700115$ PMID:19157343

70. Taboada M, Rodríguez J, Valiño C, Carceller J, Bascuas B, Oliveira J, et al. What is the minimum effective volume of local anesthetic required for sciatic nerve blockade? A prospective, randomized comparison between a popliteal and a subgluteal approach. Anesth Analg. 2006 Feb;102(2):5937. https://doi.org/10.1213/01. ane.0000189188.08679.2a PMID:16428568

71. Bang SU, Kim DJ, Bae JH, Chung K, Kim Y. Minimum effective local anesthetic volume for surgical anesthesia by subparaneural, ultrasound-guided popliteal sciatic nerve block: A prospective dose-finding study. Medicine (Baltimore). 2016 Aug;95(34):e4652. https://doi.org/10.1097/ MD.0000000000004652 PMID:27559966

72. Techasuk W, Bernucci F, Cupido T, González AP, Correa JA, Finlayson RJ, et al. Minimum effective volume of combined lidocaine-bupivacaine for analgesic subparaneural popliteal sciatic nerve block. 
Reg Anesth Pain Med. 2014

Mar-Apr;39(2):108-11.

https://doi.org/10.1097/

AAP.0000000000000051

PMID:24496169

73. Casati A, Fanelli G, KoscielniakNielsen Z, Cappelleri G, Aldegheri G, Danelli G, et al. Using stimulating catheters for continuous sciatic nerve block shortens onset time of surgical block and minimizes postoperative consumption of pain medication after halux valgus repair as compared with conventional nonstimulating catheters. Anesth Analg. 2005 Oct;101(4):1192-7. https://doi.org/10.1213/01. ane.0000167232.10305.cd PMID:16192543

74. Paqueron $X$, Narchi $P$, Mazoit JX, Singelyn $F$, Bénichou A, Macaire $P$. A randomized, observerblinded determination of the median effective volume of local anesthetic required to anesthetize the sciatic nerve in the popliteal fossa for stimulating and nonstimulating perineural catheters. Reg Anesth Pain
Med. 2009 Jul-Aug;34(4):290-5.

https://doi.org/10.1097/

AAP.0b013e3181ac9be1 PMID:19585697

75. Monahan AM, Madison SJ, Loland VJ, Sztain JF, Bishop ML, Sandhu NS, et al. Continuous Popliteal Sciatic Blocks: Does Varying Perineural Catheter Location Relative to the Sciatic Bifurcation Influence Block Effects? A Dual-Center, Randomized, Subject-Masked, Controlled Clinical Trial. Anesth Analg. 2016 May;122(5):168995. https://doi.org/10.1213/ ANE.0000000000001211 PMID:26962712

76. Hauritz RW, Pedersen EM, Linde FS, Kibak K, Børglum J, Bjoern $S$, et al. Displacement of popliteal sciatic nerve catheters after major foot and ankle surgery: a randomized controlled doubleblinded magnetic resonance imaging study. Br J Anaesth. 2016 Aug;117(2):220-7. https:// doi.org/10.1093/bja/aew172 PMID:27440634

77. Tran J, Giron Arango L, Peng
P, Sinha SK, Agur A, Chan V. Evaluation of the iPACK block injectate spread: a cadaveric study. Reg Anesth Pain Med. 2019 May;44(7):689-94. https:// doi.org/10.1136/rapm-2018100355 PMID:31061110

78. Thobhani S, Scalercio L, Elliott CE, Nossaman BD, Thomas LC, Yuratich D, et al. Novel regional techniques for total knee arthroplasty promote reduced hospital length of stay: an analysis of 106 patients. Ochsner J. 2017;17(3):233-8. PMID:29026354

79. Kim DH, Beathe JC, Lin Y, YaDeau JT, Maalouf DB, Goytizolo $E$, et al. Addition of Infiltration Between the Popliteal Artery and the Capsule of the Posterior Knee and Adductor Canal Block to Periarticular Injection Enhances Postoperative Pain Control in Total Knee Arthroplasty: A Randomized Controlled Trial. Anesth Analg. 2019 Aug;129(2):52635. https://doi.org/10.1213/ ANE.0000000000003794 PMID:30234517 